\title{
Anesthetic Management in a Patient With Glucose- 6-Phosphate Dehydrogenase Deficiency Undergoing Coblation Adenoidectomy With Septoplasty and Turbinectomy
}

\author{
Abdulrhman Saleh Dairi ${ }^{\mathrm{a}, \mathrm{b}}$, e, Ghida Dairic ${ }^{\mathrm{c}}$, Moheyeldin A. Farghaly
}

\begin{abstract}
Glucose-6-phosphate dehydrogenase (G-6-PD) is the major enzyme in the pentose phosphate pathway (PPP). The end products of this pathway are ribose-5-phosphate and nicotinamide adenine dinucleotide phosphate hydrogen (NADPH). G-6-PD deficiency is known to be the most common enzymatic deficiency in red blood cells (RBCs). Genetically, the mode of inheritance is an X-linked recessive disease. The exposure to oxidative stressors will result in hemolytic anemia including fava beans, infections, metabolic conditions such as diabetic ketoacidosis, metabolic acidosis, hyperglycemia, hypoglycemia, and hypothermia. Moreover, surgical stress and certain types of medication are known to lead to hemolytic anemia. Acute hemolytic crisis is a life-threatening situation in patients with G-6-PD deficiency. Therefore, it is extremely important to monitor the patient perioperatively. The authors present this case of successful anesthetic management in a 23 -year-old lady with G-6-PD deficiency and a previous history of acute hemolytic anemia undergoing coblation adenoidectomy with septoplasty and turbinectomy.
\end{abstract}

Keywords: Glucose-6-phosphate dehydrogenase; Deficiency; Hemolytic crisis; Medications; Hemolysis

\section{Introduction}

Glucose-6-phosphate dehydrogenase (G-6-PD) deficiency is

Manuscript submitted September 7, 2019, accepted October 1, 2019

aDepartment of Anesthesia and Pain Management, King Abdulaziz Medical City, Ministry of National Guard Health Affairs, Jeddah, Saudi Arabia

bepartment of Anesthesia and Pain Management, International Medical Center, Jeddah, Saudi Arabia

${ }^{\mathrm{c}}$ Medicine and Medical Sciences Research Center, Deanship of Scientific Research, Umm Al-Qura University, Makkah, Saudi Arabia

${ }^{\mathrm{d} D e p a r t m e n t}$ of Anesthesia, Faculty of Medicine, Ain Shams University, Cairo, Egypt

${ }^{\mathrm{e}}$ Corresponding Author: Abdulrhman Saleh Dairi, Department of Anesthesia and Pain Management, King Abdulaziz Medical City, Ministry of National Guard Health Affairs, Jeddah, Saudi Arabia.

Email: Abdulrhman_Dairi@hotmail.com

doi: https://doi.org/10.14740/jmc3371 an enzymopathy that leads to red blood cells (RBCs) hemolysis $[1,2]$. It is the most common enzymopathic disorder of RBCs $[3,4]$. It is a genetic disorder localized on the X chromosome which is inherited by an X-linked recessive pattern. Thus, it is transmitted and occurs primarily in males. Worldwide, it has been reported that more than 400 million people have been diagnosed with this disease [3-5]. The rate of new cases is associated with areas endemic for malaria. However, it is conventional in other countries [6]. A huge body of evidence considered that the G-6-PD is the most important factor in the production of antioxidants, which protecting the cells from oxidative damage [6]. Accordingly, patients with G-6-PD deficiency are highly susceptible to oxidative stressors [6]. In the previous reported cases, successful anesthetic management of a patient with G-6-PD deficiency involved the decreasing of possible oxidative stressors, and this is dependent on the type of surgery [5-7]. In our report, we present this case of successful anesthetic management in a 23 -year-old female patient with G-6-PD deficiency undergoing coblation adenoidectomy with septoplasty and turbinectomy. We also discuss the different types of medication to be used in such a patient based on reviewing the literature and our experience.

\section{Case Report}

A 23-year-old female patient (height: $150 \mathrm{~cm}$, weight: $48 \mathrm{~kg}$ ) was diagnosed as G-6-PD deficiency at the age of 7 years after a hemolytic episode, and she received a blood transfusion at that time; no other episodes were reported. She presented to the Otolaryngology-Head and Neck Surgery Clinic with symptoms of nasal obstruction, nasal tone of voice, and mouth breathing. A routine laboratory investigation was revealed: hemoglobin of $12.3 \mathrm{~g} / \mathrm{dL}$, white blood cell counts of $4.63 \times 10^{9} / \mathrm{L}, \mathrm{RBC}$ counts of $4.09 \times 10^{12} / \mathrm{L}$, and platelet of 158 $\times 10^{9} / \mathrm{L}$; all are within normal limits. Computerized tomography (CT) of paranasal sinuses showed deviated nasal septum, minimal mucosal thickening seen in the maxillary sinuses, left upper molar radicular cyst, and asymmetrical thickening of the nasopharyngeal mucosa.

The planned surgery was for coblation adenoidectomy with septoplasty and turbinectomy. Intraoperatively, a 20-gauge intravenous cannula was established, and the patient was moni- 
tored by electrocardiogram (ECG), bispectral index sensor (BIS) and the measurement of oxygen saturation $\left(\mathrm{SpO}_{2}\right)$, noninvasive blood pressure (NIBP), temperature, and followed by preoxygenation. Pre-induction vital signs revealed blood pressure of $118 / 81 \mathrm{~mm} \mathrm{Hg}$, heart rate of 82 beats per minute (bpm), $\mathrm{SpO}_{2}$ of $100 \%$, and a temperature of $36.4{ }^{\circ} \mathrm{C}$. Thereafter, general anesthesia induced with fentanyl $(100 \mu \mathrm{g})$, propofol $(150 \mathrm{mg})$, rocuronium $(30 \mathrm{mg})$, and dexamethasone $(16 \mathrm{mg})$. The patient was intubated by a direct laryngoscope with oral endotracheal Ring-Adair-Elwyn (RAE) tube (internal diameter (ID): $6.5 \mathrm{~mm}$ ) with an inflatable cuff and connected to the circuit of the anesthesia machine. Anesthesia has been maintained by the inhalational anesthetic agent sevoflurane $(2.0 \%)$ with $\mathrm{O}_{2} / \mathrm{N}_{2} \mathrm{O}$ mixture $(1.0 / 1.0 \mathrm{~L} / \mathrm{min})$. The patient was positioned supine for the surgery. Before the incision was made, cefazolin $(2 \mathrm{~g})$ was given as a prophylactic antibiotic. At $40 \mathrm{~min}$ into the surgery, we administered paracetamol $(1 \mathrm{~g})$ followed by oxycodone $(4 \mathrm{mg})$. Also, she received isotonic, crystalloid fluid (Ringer's lactate) with a total infusion of $650 \mathrm{~mL}$. The estimated blood loss was $50 \mathrm{~mL}$. Intraoperatively, the patient was vitally stable. The duration of the surgery was approximately $90 \mathrm{~min}$. At the end of the surgery, we reversed the residual neuromuscular medication with glycopyrrolate $(0.4 \mathrm{mg})$ plus neostigmine $(2.5 \mathrm{mg})$, and when the patient was fully awake, the trachea was extubated successfully. Postoperatively, oxycodone has been used as a postoperative analgesia. Moreover, we monitored the patient for any signs or symptoms of an acute hemolytic crisis. The patient was discharged on the second postoperative day (POD) after assuring the result of complete blood count (CBC) is normal.

\section{Discussion}

In the pentose phosphate pathway (PPP) which known also as phosphogluconate pathway and hexose monophosphate (HMP) shunt, the major enzyme is G-6-PD $[5,6]$. It is the first step in PPP resulting in converting glucose to ribose-5-phosphate. In our body, the only source for generating the nicotinamide adenine dinucleotide phosphate hydrogen (NADPH) is PPP [6]. In contrast, the generation of NADPH will lead to reducing cell energy by controlling the reduced glutathione within the cell [2]. Oxidative damage will be bypassed by glutathione reduction as this mechanism results in cell protection [2]. RBCs do not have another metabolic backup system as other human cells, which work to generate the intracellular NADPH $[2,8]$. Therefore, any exposure to oxidative stress will contribute to hemolytic anemia [2, 8]. Subsequently, G-6-PD deficiency is lethal in RBCs $[2,8]$. However, RBCs are vulnerable to oxidative stress, leading to hemolytic anemia [6].

Hemolysis can be happening after exposure to many factors such as fava beans, infections, metabolic conditions such as diabetic ketoacidosis, metabolic acidosis, hyperglycemia, hypoglycemia and hypothermia, and have been all over reported previously [1, 9-11]. Furthermore, certain types of medication such as antimicrobials (sulfonamides, nitrofurantoin, and chloramphenicol), non-steroidal anti-inflammatory drugs (NSAIDs), anticonvulsants, diuretics containing sulfonamide, insulin, oral hypoglycemic agents, and ranitidine, are known to lead to hemo- lytic anemia $[5,6,9-11]$. The mechanism of hemolytic anemia is due to membrane damage by oxidized hemoglobin $[5,6]$. At the same time, anesthetic drugs such as diclofenac, metoclopramide, lidocaine, methylene blue, and prilocaine are contraindicated in patients with G-6-PD deficiency $[11,12]$. While glycopyrrolate, fentanyl, sufentanil, tramadol, ketamine, propofol, thiopental, halothane, nitrous oxide, rocuronium, succinylcholine, neostigmine, bupivacaine, and heparin are reported previously as it can be administrated safely in patients with G-6-PD deficiency $[1,4,6,11]$. However, there are anesthetic agents still controversy such as sevoflurane and midazolam [6]. In our case, we administrated the inhalational agent sevoflurane, and to our experience it is safe in patients with G-6-PD deficiency. Overall, there is insufficient evidence of medications to be used in G6-PD deficiency patients, and some of them are still controversy [6]. Hence, for patients with G-6-PD deficiency, perioperative medications must be known as much as possible to avoid acute hemolytic crisis. All the administrated medications in our case were safe for a patient with G-6-PD deficiency.

Also, hemolysis can be caused by surgical stress, which has been reported in the literature [5]. Therefore, perioperative adequate analgesia will work effectively to decrease the level of stress related to the surgery [5]. We provided adequate analgesia throughout the intraoperative and postoperative interval because this surgery is painful. In such a case, our aim is to avoid acute hemolytic crisis [5]. However, acute hemolytic crisis is self-limiting and in severe rare cases granting blood transfusion [6]. After 24 to $72 \mathrm{~h}$, the hemolysis can become demonstrable in patients with risk factors $[2,6]$. Intraoperatively, reducing oxidative stress is extremely important besides monitoring and treating hemolysis [6]. During surgery, hematuria is an indicator of an acute hemolytic crisis [5]. Reaching to the diagnosis is substantial, and once that made, we have to cease the triggering agent and maintaining the urine output by infusing crystalloids as well as administration of non-sulfonamide diuretics $[5,13]$. Unfortunately, a Foley catheter was not inserted in our case because the surgical duration was short; but we recommend inserting a Foley catheter in such a patient to monitor the urine output regardless of the surgical duration. Postoperatively, the symptoms of hemolysis will be appearing clearly by 7 days after the hemolysis, which include headache, fatigue, tachycardia, cyanosis, dyspnea, lethargy, lumbar pain, abdominal pain, splenomegaly, hemoglobinuria and scleral icterus $[3,6]$. To monitor the need for blood transfusion, CBC must be done daily after an episode of acute hemolytic crisis [5]. Microscopic examination of the peripheral blood smear will show RBC fragments such as reticulocytes, schistocytes, and Heinz bodies. Other lab investigations that can be done are lactate dehydrogenase and unconjugated bilirubin which will be increased, whereas haptoglobin levels will decrease $[2,6]$. Additionally, urobilinogen and hemosiderin can be detected in the urine $[2,6]$. Also, we can use the direct Coombs test to rule out an immunological reaction $[2,6]$.

\section{Conclusions}

From an anesthetic point of view, acute hemolytic crisis is correlated with different triggers as we previously mentioned. Out 
of these triggers, there are particular types of medication we have to avoid as much as possible in patients with G-6-PD deficiency. Moreover, monitoring the patient perioperatively for such a crisis is extremely important, which includes surgical stress, because acute hemolytic crisis is a life-threatening situation in these patients.

\section{Acknowledgments}

None to declare.

\section{Financial Disclosure}

None to declare.

\section{Conflict of Interest}

None to declare.

\section{Informed Consent}

Obtained.

\section{Author Contributions}

Abdulrhman Saleh Dairi is the first author involved in the literature review and preparation of the manuscript (initial and revised versions). Moheyeldin A. Farghaly and Ghida Dairi are involved in the critical revision of all versions of the manuscript.

\section{References}

1. Fodinger AM, Kammerlander C, Luger TJ. Ultrasoundguided regional anesthesia in a Glucose-6-Phosphate Dehydrogenase (G6PD)-deficient geriatric trauma patient. Geriatr Orthop Surg Rehabil. 2012;3(4):147-149.

2. Elyassi AR, Rowshan HH. Perioperative management of the glucose-6-phosphate dehydrogenase deficient patient: a review of literature. Anesth Prog. 2009;56(3):86-91.

3. Takahashi N, Ogawa T, Wajima Z, Omi A. Dexmedetomidine-based intravenous anesthesia of a pediatric patient with glucose-6-phosphate dehydrogenase (G6PD) deficiency: A case report. Medicine (Baltimore). 2017;96(21):e6986.

4. Glucose-6-phosphate dehydrogenase deficiency. WHO Working Group. Bull World Health Organ. 1989;67(6):601-611.

5. Valiaveedan S, Mahajan C, Rath GP, Bindra A, Marda MK. Anaesthetic management in patients with glucose6-phosphate dehydrogenase deficiency undergoing neurosurgical procedures. Indian J Anaesth. 2011;55(1):68-70.

6. Cho H, Young Lee S, Hee Kim G, et al. Anesthetic management of a patient with glucose-6-phosphate dehydrogenase deficiency undergoing robot-assisted laparoscopic surgery. A case report. Anesthesia and Pain Medicine. 2017;12:243-246.

7. Dogra N, Puri GD, Rana SS. Glucose-6-phosphate dehydrogenase deficiency and cardiac surgery. Perfusion. 2010;25(6):417-421.

8. Cappellini MD, Fiorelli G. Glucose-6-phosphate dehydrogenase deficiency. Lancet. 2008;371(9606):64-74.

9. Hou L, Lin S, Meng Z, Zhang L, Liu Z, Luo X, Liang L. Young type 1 diabetes mellitus (T1DM) patient with glucose-6-phosphate dehydrogenase deficiency occurring hemolysis: A case report. Biomedical Research. 2017;28(16):6994-6996.

10. Carette C, Dubois-Laforgue D, Gautier JF, Timsit J. Diabetes mellitus and glucose-6-phosphate dehydrogenase deficiency: from one crisis to another. Diabetes Metab. 2011;37(1):79-82.

11. Sharma V, Verma S, Bhatia PK, Sethi P. Propofol infusion in an infant with glucose-6-phosphate dehydrogenase deficiency. J Anaesthesiol Clin Pharmacol. 2018;34(1):136137.

12. Bubp J, Jen M, Matuszewski K. Caring for Glucose6-Phosphate Dehydrogenase (G6PD)-deficient patients: implications for pharmacy. P T. 2015;40(9):572-574.

13. Balderia PG, Wongrakpanich S, Patel M, Stanek M. Healing the orphaned heart: heart failure in a patient with glucose-6-phosphate dehydrogenase deficiency. BMJ Case Rep. 2015;2015. 